\title{
THE OBSTRUCTION TO THE LOCALIZABILITY OF A MEASURE SPACE ${ }^{1}$
}

\author{
BY F. E. J. LINTON \\ Communicated by P. R. Halmos, November 9, 1964
}

This paper, an outgrowth of the author's doctoral dissertation, ${ }^{2}$ presents a necessary and sufficient condition, of a cohomological nature, for a measure space to be localizable in the sense of Segal. ${ }^{3}$ In order to state the main theorem, we must fix some terminology and establish some notation.

1. Definitions. ${ }^{4}$ A measure space $(X, R, m)$ consists of a set $X$, a boolean ring $R$ of subsets of $X$, and a finite, nonnegative, finitely additive measure $m$ on $R$ subject to the requirement:

$$
\begin{aligned}
& \left\{E_{n} \in R(n=1,2, \cdots), E_{n} \cap E_{k}=\varnothing(n \neq k),\right. \\
& \left.\quad \Sigma_{n} m\left(E_{n}\right)<\infty, E=\bigcup_{n} E_{n}\right\} \Rightarrow\left\{E \in R \text { and } m(E)=\sum_{n} m\left(E_{n}\right)\right\} .
\end{aligned}
$$

If $(X, R, m)$ is a measure space, a subset $K$ of $X$ is measurable if $K \cap E \in R$ whenever $E \in R$; it is null if it is measurable and $m(K \cap E)$ $=0$ whenever $E \in R$. The measure ring $\mathfrak{T}$ of the measure space $(X, R, m)$ is the quotient of the (sigma ring of) measurable sets by the (sigma ideal of) null sets. A measure space is localizable if its measure ring is complete as a partially ordered set.

2. Let $(X, R, m)$ be a measure space. Consistent use will be made of the following notation:

$I$ : the ideal of sets $K \in R$ for which $m(K)=0$;

$M_{1}$ : the sigma ring of measurable sets;

$X_{R}$ : the set $\cup R \in M_{1}$;

$M$ : the principal ideal of $M_{1}$ determined by $X_{R}$;

$N_{1}$ : the sigma ideal of null sets in $M_{1}$;

$N$ : the sigma ideal of null sets in $M$, i.e., $M \cap N_{1}$;

\footnotetext{
1 Research supported partly by the Air Force Office of Scientific Research under contract AFOSR 520-64 and partly by the National Science Foundation under grant NSF GP-2432. The author takes this opportunity to thank I. Kaplansky and D. M. Topping for their helpful interest in this work. Further details and related results will appear elsewhere.

${ }^{2}$ F. E. J. Linton, The functorial foundations of measure theory, Columbia Univ., New York, 1963.

3. E. Segal, Equivalences of measure spaces, Amer. J. Math. 73 (1951), 275-313.

4 This is merely a restatement, for the convenience of the reader, of parts of Definitions 2.1, 2.2, 2.4, and 2.6 in the cited work of Segal. Incidentally, our rings need not have unit elements.
} 
$r$ : the countably additive measure on $M_{1}$ defined by $r(K)$ $=\sup _{E \in R} m(K \cap E)$.

3. The following observations are easily verified. ${ }^{5}$

(3.1) For $K \in M_{1}, r(K)=0$ iff $K \in N_{1}$.

(3.2) Every subset of $X$ disjoint from $X_{R}$ is null.

(3.3) $r$ and $m$ take the same values on $R$.

(3.4) $\Re \cong M_{1} / N_{1} \cong M / N$.

4. The obstruction. Let $J$ be an ideal in a boolean ring $A$. Since the intersection of an element of $J$ with an element of $A$ is again an element of $J$, we may consider $J$ as a sub- $A$-module of the $A$-module $A$; in consequence, we may also think of the quotient ring $A / J$ as an $A$-module-this $A$-module structure on $A / J$ is the same as that induced from the natural $(A / J)$-module structure by change of rings using the canonical projection $A \rightarrow A / J$. Let $d_{A, J}$ denote the connecting homomorphism ${ }^{6}$

$$
d_{A, J}: \operatorname{Hom}_{A}(A, A / J) \rightarrow \operatorname{Ext}_{A}^{1}(A, J)
$$

between the group of $A$-module homomorphisms $A \rightarrow A / J$ and the group of $A$-module extensions $0 \rightarrow J \rightarrow ? \rightarrow A \rightarrow 0$. We call $d_{A, J}$ the $o b$ struction to the localizability of the boolean ring $A$ over its ideal $J$. If $d_{A, J}=0$, we say $A$ is localizable over $J$.

5. Main theorem. The measure space $(X, R, m)$ is localizable if and only if the obstruction $d_{R, I}$ to the localizability of $R$ over $I$ vanishes.

It is to be noted that no question of higher obstructions arises.

6. The ring $\beta A$. The proof of Theorem 5 depends on information regarding the largest boolean ring $\beta A$ containing a given boolean ring $A$ as a dense ideal. ${ }^{7}$ The uniqueness of $\beta A$ is due to the fact that its Stone space must be the Stone-Čech compactification of the Stone space of $A$. Its existence is demonstrated by proving that the clopen sets in that compactification have the desired property. Alternate equivalent descriptions of $\beta A$ are:

(6.1) $\beta A$ is the ring of clopen sets in the Stone space of $A$ ( $A$ appears as the ring of compact open sets);

(6.2) if $A$ is represented as a ring of subsets of a set $Z$, then $\beta A \cong\{Y \mid Y \subseteq \cup A, Y \cap a \in A$ for all $a \in A\}$;

s Consult Segal, op. cit., for proofs of (3.1) and (3.3); the proof of (3.2) is immediate, and (3.4) follows from the rest.

- Cf. S. Mac Lane, Homology, p. 74, Springer, Berlin, 1963.

${ }^{7}$ Some of the material presented in $\$ 6$ is contained in $\$ 1.10$ of the author's dissertation. 
(6.3) $\beta A \cong \mathrm{Hom}_{A}(A, A)$ (the ring of $A$-module endomorphisms of A);

(6.4) $\beta A$ is the inverse limit of the inverse system

$$
\left(\left\{A_{a}\right\}_{a \in A}, \quad\left\{p_{a, b}: A_{a} \rightarrow A_{b}\right\}_{a \geq b}\right)
$$

of all principal ideals $A_{a}=\{x \mid x \leqq a\}$ of $A$, where $p_{a, b}(x)=x \wedge b$.

7. The main lemma. If $J$ is an ideal in the boolean ring $A$, the canonical projection $A \rightarrow A / J$ induces three maps

$$
\begin{gathered}
\operatorname{Hom}_{A}(A, A / J) \leftarrow \operatorname{Hom}_{A}(A / J, A / J) \\
\operatorname{Hom}_{A}(A, A) \rightarrow \operatorname{Hom}_{A / J}(A / J, A / J)
\end{gathered}
$$

by covariant composition, contravariant composition, and change of rings, respectively. The second and third maps are isomorphisms. The indicated inverse composite is easily seen to be a unitary ring homomorphism; this, when combined with the representations (6.3) of $\beta A$ and $\beta(A / J)$, yields a distinguished map

$$
\beta A \rightarrow \beta(A / J),
$$

about which the essential information is recorded in the lemma below. The first statement of this lemma is clear from the discussion above; the remaining statements depend only upon the exactness ${ }^{8}$ of the sequence

$$
0 \rightarrow \operatorname{Hom}_{A}(A, J) \rightarrow \operatorname{Hom}_{A}(A, A) \rightarrow \operatorname{Hom}_{A}(A, A / J) \stackrel{d_{A}, J}{\longrightarrow} \operatorname{Ext}_{A}^{1}(A, J) .
$$

(7.3) MAIN LEMma. The map (7.2) is a boolean homomorphism. In the representation (6.3), its kernel is given by $\operatorname{Hom}_{A}(A, J)$; using (6.2), instead, its kernel is the family

$$
\tilde{J}=\{Y \mid Y \subseteq \cup A, Y \cap a \in J \text { for all } a \in A\} .
$$

Moreover, the induced monomorphism $\beta A / \tilde{J} \rightarrow \beta(A / J)$ is an isomorphism if and only if $A$ is localizable over $J$.

8. Although (7.3) is the main tool used in the proof of Theorem 5 , a few simple observations must be made before it can successfully be applied. Namely, let $(X, R, m)$ be a measure space. Then:

(8.1) $M \cong \beta R$ (consequence of (6.2));

(8.2) $N \cong I$ (consequence of (7.3));

(8.3) $\mathfrak{T} \cong \beta R / \tilde{I}$ (consequence of (3.4), (8.1), (8.2));

- Cf. Mac Lane, loc. cit. 
(8.4) $R / I \subseteq \dddot{M} \subseteq \beta(R / I)$ (consequence of (3.3), (7.3), (8.3));

(8.5) $\beta(R / I)$ is complete (consequence of (6.4), the completeness of each principal ideal in $R / I$, and the fact that each map in the inverse system (6.4) is a complete homomorphism).

9. Proof of Theorem 5. According to Lemma (7.3), when we take into account (8.3) and (8.4), a necessary and sufficient condition for $R$ to be localizable over $I$ is that the inclusion $\Re \subseteq \beta(R / I)$ be the identity. If, indeed, this is the identity, (8.5) assures that $\mathfrak{T}$ is complete, so that $(X, R, m)$ is localizable. If, conversely, $(X, R, m)$ is localizable, $\mathscr{T}$ is complete, and since complete boolean rings are injective, ${ }^{9}$ the inclusion $\mathfrak{T} \subseteq \beta(R / I)$ admits a retraction $p: \beta(R / I) \rightarrow \mathscr{T}$. In order to prove $\mathbb{T}=\beta(R / I)$, it suffices to know that this retraction is one-one. So let $b \in \beta(R / I)$, and assume $b \neq 0$. Then $b$ contains a nonzero element $a \in R / I$, and, by (8.4), $p(a) \neq 0$ (indeed, $p(a)=a)$. But $p(b) \geqq p(a)$, since $b \geqq a$, and so $p(b) \neq 0$. Thus $p$ is one-one, $\mathfrak{T}=\beta(R / I)$, and the proof is complete.

10. Localizability and the dual of $L_{1}$. Theorem 5 can be used to deliver a quick and revealing proof of Segal's theorem ${ }^{10}$ that the measure space $(X, R, m)$ is localizable if and only if the usual "integral of the product" map from the Banach space $L_{\infty}$ of (essentially) bounded $M_{1}$-measurable functions mod $N_{1}$-measurable functions to the dual of the space $L_{1}=L_{1}(X, R, m)$ is an isomorphism. For by an extension of a theorem ${ }^{11}$ of Sikorski, $L_{\infty}$ is isomorphic with the space ${ }^{12}$ of bounded Carathéodory functions on $M_{1} / N_{1} \cong \mathfrak{M} \cong \beta R / \tilde{I}$. On the other hand, the dual of the space $L_{1}$ is ${ }^{13}$ the space of bounded Carathéodory functions on $\beta(R / I)$, which, because $\beta R / \tilde{I} \subseteq \beta(R / I)$, contains $L_{\infty}$ as an isometrically embedded subspace. Consequently, $L_{\infty}$ is the dual of $L_{1}$ if and only if these two spaces of Carathéodory functions coincide, and this, in turn, is the case if and only if $\beta R / \mathcal{I}$ and $\beta(R / I)$ coincide, i.e., using Theorem 5 and Lemma (7.3), if and only if $(X, R, m)$ is localizable.

The University of Chicago AND WESLEYAN UNIVERSITY

- Cf. P. R. Halmos, Injective and projective boolean algebras, Proc. Sympos. Pure Math., Vol. 2, pp. 114-122, Amer. Math. Soc. Providence, R. I., 1961.

${ }^{10}$ Segal, op. cit.

${ }^{11}$ R. Sikorski, Boolean algebras, Proposition 32.5, Springer, Berlin, 1960.

${ }^{12} \mathrm{Cf}$. C. Goffman, Remarks on lattice-ordered groups and vector lattices. I. Carathéodory functions, Trans. Amer. Math. Soc. 88 (1958), 107-120.

${ }^{13}$ Theorem (2.5.10) of the author's dissertation. 